Am. J. Trop. Med. Hyg., 71(Suppl 2), 2004, pp. 136-140

Copyright $@ 2004$ by The American Society of Tropical Medicine and Hygiene

\title{
THE BURDEN OF MALARIA EPIDEMICS AND COST-EFFECTIVENESS OF INTERVENTIONS IN EPIDEMIC SITUATIONS IN AFRICA
}

\author{
EVE WORRALL, AAFJE RIETVELD, AND CHARLES DELACOLLETTE \\ Health Policy Unity, Department of Public Health and Policy, London School of Hygiene and Tropical Medicine, London, United \\ Kingdom; Malaria Control Department, World Health Organization, Geneva, Switzerland
}

\begin{abstract}
Attempts to quantify the epidemiologic and economic burden of malaria have so far neglected to specifically address the burden of epidemic malaria. Moreover, the data on the effectiveness and cost-effectiveness of interventions in epidemics is extremely limited. Answering such key questions in an epidemic prone context is more challenging than doing so in endemic areas. Using the limited data available, we estimate that in Africa, there are more than 12 million malaria episodes and 155,000-310,000 malaria deaths per year attributable to epidemics if control options are not implemented or well timed, which is equivalent to some $4 \%$ of estimated annual malaria cases worldwide and $12-25 \%$ of estimated annual worldwide malaria deaths, including up to $50 \%$ of the estimated annual worldwide malaria mortality in persons $>15$ years of age. The possible economic impact of malaria epidemics is described and the limited evidence on the effectiveness and cost-effectiveness of interventions in areas of low or seasonal transmission is reviewed.
\end{abstract}

\section{INTRODUCTION}

Plasmodium falciparum malaria is a life-threatening disease for individuals with low immunity. Communities that are not normally exposed to high rates of malaria transmission are therefore vulnerable to explosive epidemics that can cause high case fatality rates among all age groups. Epidemics can be defined by their main causal factors and in this paper we concentrate on those triggered predominately by climate or weather.

In spite of their severity, research on malaria epidemics is limited. Estimates of the population at risk of weather or climate dependent malaria epidemics in Africa are contradictory which makes estimation of the epidemiologic burden of epidemics problematic. Very little is known about the economic burden of epidemics. The current lack of accurate documentation of the epidemic malaria burden and its economic impact means that planning and targeting of resources is uncertain and epidemics may not receive the attention or resources that they may deserve. In particular, the severe dearth of information regarding the cost-effectiveness of prevention and control interventions in epidemic prone contexts means that policy makers cannot make resource allocation decisions based on sound evidence.

In this paper, we attempt to bring together the limited quantitative data on the epidemiology of epidemic malaria and use this in conjunction with estimates of the population living in malaria-risk areas to quantify the epidemiologic burden of malaria epidemics. The main difficulties in quantifying the burden of epidemic malaria are discussed along with the resulting implications for measuring the economic burden of malaria epidemics. The evidence relating specifically to the economic burden of epidemics and the cost and effectiveness of interventions in epidemic settings is then reviewed to identify gaps and priorities for future research.

\section{MATERIALS AND METHODS}

To estimate the epidemiologic burden of malaria epidemics, we first present a definition of the types of malaria epidemics and identify those included in this analysis. On the basis of this definition, we examine available estimates of the population at risk of epidemics. Estimates of the frequency of occurrence of epidemics are obtained from the literature and applied to our chosen at risk population estimate. We then go on to examine the possible consequences of these epidemics in terms of morbidity and mortality using available figures and place our results in the context of estimated annual worldwide malaria mortality.

A forthcoming review has identified that quantitative estimates of the economic burden of epidemic malaria are presently lacking. ${ }^{1}$ We therefore describe the levels at which the economic impact of epidemics may be felt and provide some quantitative evidence from the gray literature where available.

A literature review was carried out to identify published studies on the effectiveness and cost-effectiveness of malaria interventions in areas of low, seasonal, or epidemic malaria transmission. The results of this review are presented and discussed.

Definition of epidemics. Malaria epidemics can be categorized on the basis of their main causal factors ${ }^{2}$ (Figure 1). First, they may be caused by climate anomalies (for example excessive or prolonged rainfall or unusual increases in temperature), often occurring in arid or semi-arid zones where the human population is relatively or completely nonimmune. Second, in other areas, malaria transmission may be strongly seasonal with some degree of inter-annual variation caused by normal variations in climatology, perhaps as the result of meteorologic phenomena such as strong El Niño Southern Oscillation events that are commonly associated with drought or floods and higher than average temperature. ${ }^{3}$ Third, epidemics may occur during complex emergency situations where normal malaria transmission might be exacerbated by sudden population movements, war, and political instability. A neglect or breakdown in control activities may also give previously controlled malaria the opportunity to reemerge, leading to a subsequent increase in transmission and endemicity lasting for several transmission seasons. ${ }^{2}$ Whatever their cause, the impact of malaria epidemics is greatest when they follow periods of drought and famine when populations are most vulnerable. ${ }^{4}$

In this paper, we estimate the burden of the first two types of epidemics described previously. These can be broadly categorized as occurring due to abnormal meteorological conditions and as such have been described as occurring in "quasiregular cycles." 2 The burden of epidemics occurring in com- 


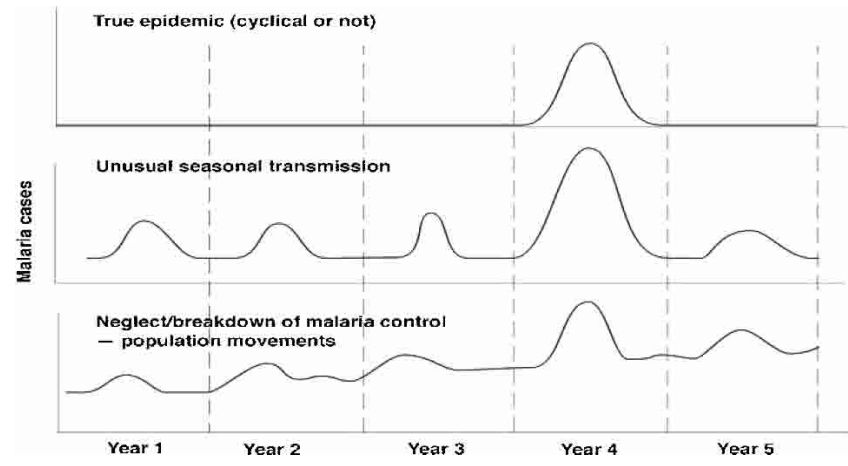

FIGURE 1. Classification of major malaria epidemic types. ${ }^{25}$ The top panel shows the pattern of cases in a true epidemic. The middle panel shows how cases may occur in epidemics caused by unusual seasonal transmission. The bottom panel shows epidemics caused by a neglect or breakdown in malaria control and/or population movements, both of which may or may not be linked to complex emergencies.

plex emergencies or as a result of a breakdown in control activities is excluded since they are not obviously cyclical or regular in nature and therefore cannot be estimated using this methodology.

Population at risk. The duration of the malaria transmission season can be taken as a rough proxy for the likelihood of epidemics occurring. Generally, as the transmission season gets shorter, the risk of epidemics increases. In Africa, the areas with a shorter transmission season tend to be located across the Sahelean belt, down through the horn of Africa into east Africa and throughout southern Africa.

Overall estimates of the population at risk of climate dependent malaria epidemics in Africa vary depending on the method and data used. Snow and others estimated that the population exposed to epidemic malaria risk in Africa for 1995 was approximately 74 million. ${ }^{5}$ A World Health Organization (WHO) estimate determined in 1996 by country expert opinion based on historical malaria incidence data and knowledge of risk factors estimated the same figure to be approximately 112 million. ${ }^{6}$ This figure has recently been recalculated using United Nations population data for 2001, and the resulting estimate is 124.7 million (Kawano M, meeting of the Roll Back Malaria Technical Support Network on Epidemics, Leysin, Switzerland, December 2003). Testing and validation of approaches to measuring the population at risk of malaria epidemics are planned. ${ }^{7}$ However, for the purpose of this study, we chose to use the updated WHO estimate since it uses more recent population data. Table 1 provides a country-by-country breakdown of the population estimated to live in areas prone to or at risk of malaria epidemics throughout Africa according to these calculations.

Epidemiologic burden of malaria epidemics. Malaria epidemics can recur in cycles of varying length caused by different precipitating factors. It is therefore difficult to calculate a precise average annual epidemiologic burden of disease. However, most weather or climate driven epidemic follow cycles of 2-7 years reflecting the periodicity of abnormal meteorologic conditions. ${ }^{2}$

It has been estimated that each person will have approximately 0.5 malaria episodes per epidemic. ${ }^{2}$ Assuming (conservatively) that epidemics occur on average every five years, ${ }^{8}$ this gives an estimated 0.1 episodes of malaria per
TABLE 1

Estimated population at risk of malaria epidemics ${ }^{26}$

\begin{tabular}{lrcr}
\hline \multicolumn{1}{c}{ Country } & $\begin{array}{c}\text { Total } \\
\text { population }\end{array}$ & $\begin{array}{c}\text { Estimated } \\
\text { percentage of } \\
\text { population at } \\
\text { risk for epidemics }\end{array}$ & $\begin{array}{c}\text { Estimated } \\
\text { number of } \\
\text { people at risk } \\
\text { for malaria } \\
\text { epidemics }\end{array}$ \\
\hline Angola & $13,527,000$ & 35 & $4,734,450$ \\
Botswana & $1,553,000$ & 40 & 621,200 \\
Burundi & $6,501,000$ & 50 & $3,250,500$ \\
Cape Verde & 436,000 & 50 & 218,000 \\
Chad & $8,134,000$ & 15 & $1,220,100$ \\
DR* Congo & $52,521,000$ & 10 & $5,252,100$ \\
Eritrea & $3,815,000$ & 60 & $2,289,000$ \\
Ethiopia & $64,458,000$ & 50 & $32,229,000$ \\
Kenya & $31,292,000$ & 25 & $7,823,000$ \\
Madagascar & $16,436,000$ & 50 & $8,218,000$ \\
Mali & $11,676,000$ & 20 & $2,335,200$ \\
Mauritania & $2,746,000$ & 40 & $1,098,400$ \\
Mauritius & $1,170,000$ & 40 & 468,000 \\
Mozambique & $18,644,000$ & 20 & $3,728,800$ \\
Namibia & $1,787,000$ & 40 & 714,800 \\
Niger & $11,226,000$ & 20 & $2,245,200$ \\
Rwanda & $7,948,000$ & 50 & $3,974,000$ \\
Senegal & $9,661,000$ & 10 & 966,100 \\
Somalia & $9,156,000$ & 50 & $4,578,000$ \\
South Africa & $43,791,000$ & 5 & $2,189,550$ \\
Sudan & $31,809,000$ & 50 & $15,904,500$ \\
Swaziland & 937,000 & 40 & 374,800 \\
Tanzania & $35,964,000$ & 25 & $8,991,000$ \\
Uganda & $24,022,000$ & 10 & $2,402,200$ \\
Zambia & $10,648,000$ & 50 & $5,324,000$ \\
Zimbabwe & $12,851,000$ & 28 & $3,598,280$ \\
Total & $432,709,000$ & & $124,748,180$ \\
\hline * DR Detal & & &
\end{tabular}

* DR $=$ Democratic Republic.

person per year for the population living in areas at risk of epidemics. Multiplying this by the population living in areas at risk of malaria epidemics in gives an estimated 12.4 million malaria episodes per year attributable to epidemics in Africa, which is equivalent to some $4 \%$ of annual malaria cases worldwide. ${ }^{9}$

Since epidemics occur in populations with little or no immunity, an estimated $5 \%$ of cases may progress to severe malaria, which has a case fatality rate of approximately $10 \%$ in reasonably well-equipped referral facilities. ${ }^{10}$ In epidemic situations, facilities are likely to be under equipped, over used, and under staffed. Staff may be caught unprepared and with insufficient drugs, and patients may be more vulnerable due to pre-existing malnutrition. All these factors contribute to an increased case fatality rate for severe malaria (defined according to $\mathrm{WHO}$ criteria $^{11}$ ) of perhaps up to $25-50 \%$ in epidemic situations.

Applying these figures to the 12.4 million epidemic-related episodes per year results in an estimated 620,000 severe cases per year of which up to $155,000-310,000$ may result in death. This is the equivalent of $12-25 \%$ of the annual worldwide malaria deaths, including up to $50 \%$ of the estimated annual worldwide malaria mortality in persons $>15$ years of age.

In addition to the components of the direct epidemiologic burden of malaria epidemics described earlier, there is likely to be an increase in the incidence of low birth weight babies and other complications arising from malaria in pregnancy following epidemics. For example, during the malaria epidemic in Sri Lanka in 1934-1935, a case fatality rate of $13 \%$ was recorded among pregnant women and the rate of fetal loss or neonatal death was almost $70 \% .^{12,13}$ In an unstable 
transmission area on the northwestern border area of Thailand, Plasmodium falciparum malaria was the main cause of maternal deaths before the institution of antenatal clinic programs (White $\mathrm{N}$, unpublished data), and pregnant women were found to be two times more likely to develop severe malaria than non-pregnant women. ${ }^{14}$ An epidemic in Rwanda in 1998 led to a four-fold increase in malaria admissions among pregnant women and a five-fold increase in maternal deaths due to malaria. ${ }^{13}$ A study in the highlands of Madagascar found that although a greater percentage of low birth weights was recorded at lowland clinics than at highland ones ( $17.1 \%$ and $9.7 \%$, respectively), the relative risk of low birth weight linked to placental infection was found to be far greater in the highlands than in the lowlands. ${ }^{15}$ In Ethiopia, placental parasitemia was associated with an increased risk of prematurity (relative risk $=3.9$ ) and a seven-fold increased risk of stillbirths in areas of unstable transmission. ${ }^{16}$

Economic burden of malaria epidemics. The major problem in estimating the economic burden of malaria epidemics is that it is difficult to estimate the economic impact of the problem without a sound understanding of the epidemiologic burden. However, in addition to this, fundamental questions relating to the economic impact of epidemics at all levels of the economy remain unanswered.

There is no recent evidence from Africa on the macroeconomic impact of epidemics, although it is likely that epidemics, or the possibility of epidemics, may deter or reverse economic growth and development. For example, in the private sector in highland tea or coffee estates, the impact of malaria epidemics on the productivity, profitability, and growth of companies remain unquantified, although recently work has begun to quantify the cost of malaria epidemics on a sugar estate and orange plantation in Ethiopia (Ministry of Health, Ethiopia). In the public sector, epidemics may impose large political and opportunity costs on governments or Ministries of Health, who may have to divert funds from other areas to deal with large-scale epidemic emergencies. A 1996-1997 malaria epidemic in one district of Zimbabwe was estimated to add approximately US\$290,000 to the direct costs of malaria control to the Ministry of Health alone, when compared with costs in the previous, non-epidemic, year (Worrall E, Hongoro $\mathrm{C}$, unpublished data). Aside from this estimate, the direct and indirect costs which malaria epidemics impose on governments remain largely unquantified.

The costs of epidemics to communities and households are not well documented. Investment and community level development may be undermined or wiped out by epidemics. It may also be deterred completely simply by the threat of epidemics occurring. Community structures and coping strategies may be stretched to the limit or breakdown in serious epidemic situations. At the household or individual level, a lack of resources to cope with or mitigate the situation may cause additional suffering and force negative economic decisions such as the panic sales of assets and increasing debts to pay for care or funerals of adults as well as children. The threat of epidemics may also influence decisions relating to crop choice, family planning, and the level of investment. The long-term effects of these decisions on individual and household welfare are unknown.

Review of effectiveness and cost-effectiveness of interventions in epidemics. The evidence relating to the effectiveness and cost-effectiveness of interventions in areas of unstable, low, or epidemic malaria transmission is extremely limited and at times mixed or contradictory.

The accuracy of rainfall and temperature as predictors of malaria epidemics in different epidemiologic and geographical settings still needs to be established. However, effective epidemic prediction tools, or "malaria early warning systems" (MEWS), have the potential to improve the timeliness and thereby cost-effectiveness of interventions such as indoor residual house spraying, ${ }^{3}$ and possibly, other preventive or control interventions associated with epidemic preparedness such as drug/supplies stock maintenance. However, the economic benefits of malaria early warning systems have so far only been evaluated hypothetically using a modeling approach. (Worrall E, 2001. An Economic Evaluation of Malaria Early Warning Systems in Africa: A Population Dynamic Modelling Approach. $\mathrm{PhD}$ Thesis, Liverpool, United Kingdom: University of Liverpool). There is currently no evidence regarding the cost or effectiveness of early detection indicators such as monitoring unusual increases in malaria patients, blood slide positivity, or demand for blood transfusions. It is likely that these options would be relatively low cost, but their impact on potential epidemics would be limited by the willingness and capacity of the system to respond to such indicators with appropriate interventions within the given lead time.

The evidence on insecticide-treated nets (ITNs) compared with indoor residual spraying (IRS) is mixed when comparing the cost-effectiveness in areas of low or seasonal transmission. In a low transmission area of South Africa, ITNs were found to be more effective but more expensive. ${ }^{17}$ In contrast to this result, IRS was found to be both more effective and cheaper than ITNs in an area of seasonal transmission in the Kenyan highlands. ${ }^{18}$ As a tool for preventing anemia in pregnancy, ITNs were found to be ineffective in a high transmission area of Kenya, but did reduce anemia in an area of low transmission on the Thailand/Burma (Myanmar) border. ${ }^{19,20}$ Intermittent preventive treatment for the prevention of malaria and anemia in pregnancy has been found to be effective in high transmission areas in Africa, ${ }^{21}$ but there are no data on its effectiveness in low transmission areas and during epidemics. Weekly chemoprophylaxis has been found to be effective for prevention of malaria in pregnancy in a low transmission area in Thailand, ${ }^{22}$ and studies on its effectiveness in a low transmission area of Madagascar are ongoing. ${ }^{15}$ Data on the costeffectiveness (as opposed to effectiveness) of these interventions in unstable transmission areas are not available.

\section{DISCUSSION}

Estimating the epidemiologic burden of malaria epidemics is more challenging than estimating or measuring the burden of endemic malaria for a number of reasons. First, different definitions are used to describe epidemics and these may vary according to epidemiologic settings. Currently, no standardized thresholds for the declaration of epidemics have been agreed upon since they strongly depend on local epidemiologic situations. Efforts are ongoing to field test and validate epidemic thresholds to identify unusual deviations from normal figures in various epidemiologic settings/conditions especially in Africa. ${ }^{7}$

Second, the different types of epidemics mean that in some instances, their impact will be impossible to estimate in an 
accurate manner. For example, in complex emergency situations, it may be difficult to separate morbidity and mortality caused by malaria from that caused by other factors. Attempts have been made by some non-governmental organizations (e.g., International Rescue Committee ${ }^{23}$ and Médicins sans Frontières ${ }^{24}$ ) to quantify the additional impact of civil war and emergencies on mortality, but only in endemic settings.

Third, there are many potential problems relating to insufficient or unreliable data. For example, surveillance data on epidemics is often not specifically collected or recorded because epidemics may be over before health services have had time to intervene, or because in severe situations, reporting procedures may break down. Epidemics also tend to affect marginalized and underserved populations that may be outside of the usual reporting systems. In some instances, more cases will go unreported than usual because families may be unable to attend facilities for financial or health reasons, both of which will be compounded in a severe epidemic. In other instances, a free-drugs policy in government health services during times of epidemics may attract many of the patients who would normally have gone to private sector providers, thus affecting comparability of such data. Proper postepidemic assessment exercises, which would address many of these issues, are rarely undertaken.

Fourth, the effects of malaria in pregnancy in epidemic situations that will continue months after the epidemic is considered to be over are only just beginning to be understood and consequently may be underestimated or ignored completely.

Finally, epidemics are heterogeneous in nature; the size of the population affected, the frequency and duration of occurrence, and the severity of epidemic outbreaks will all vary depending on local conditions and response capacity. This makes it difficult to extrapolate burden estimates from the limited data that are currently available.

Most epidemics by their nature are cyclical and are predictable only on a probabilistic basis, which makes it difficult to plan in advance for a study on the cost-effectiveness of an intervention in an epidemic situation. Any analysis must therefore be done on an opportunistic or retrospective basis (post-assessment field analysis exercise), or through the use of modeling techniques. Measuring effectiveness will be particularly problematic because pre-intervention and postintervention comparison is not appropriate since the number of malaria cases may not be constant or follow an annual pattern. It is also likely to be difficult to find suitable control areas for comparison between interventions. Establishing the counter factual (what would have happened with or without the intervention in question) is also difficult in epidemic situations because estimates of effectiveness may not accurately capture the transmission reducing effects of an intervention. This is of critical importance in bringing epidemics under control. Moreover, available evidence on effectiveness of particular interventions is likely to be based on the effectiveness of interventions in endemic settings, which may not be comparable. Finally, even if an economic evaluation such as a costeffectiveness analysis can be satisfactorily carried out, the transferability of the results to other settings will be limited by the unique nature of many epidemics and the settings in which they occur.

\section{CONCLUSION}

The epidemiologic and economic impact of epidemic malaria is likely to be different from that of endemic malaria. To move forward with accurately estimating the burden of epidemic malaria, it must be recognized that the evidence on the economic impact, effectiveness, and cost-effectiveness of interventions obtained from endemic settings is not necessarily applicable to areas prone to unstable or epidemic transmission. While substantial progress has been made over recent years, thanks to the support of the Roll Back Malaria initiative, in estimating the burden of malaria, the human and economic burden of malaria epidemics remains largely unquantified, although our estimates show that it is clearly significant: epidemic malaria in Africa alone may be causing up to $25 \%$ of annual malaria mortality worldwide, including up to $50 \%$ of adult malaria deaths. In particular, the effects of malaria in pregnancy during and following epidemics have been neglected.

To complement these preliminary estimates, there is an urgent need to carry out high-quality, multidisciplinary research into the epidemiologic and socioeconomic impact of malaria epidemics. Routine data collection, reporting, and post-epidemic assessment activities must be strengthened and expanded to generate data on the burden of epidemics. This data can then be collated and triangulated with broad quantitative estimates such as that presented in this paper to generate reliable and useful data on malaria epidemics. The use of detailed post-epidemic assessments including costeffectiveness analyses of interventions and other innovative techniques such as simulation modeling will be necessary to establish an evidence base on the cost-effectiveness of interventions to control malaria epidemics in a variety of epidemiologic and operational settings. This evidence is required to support planning and policy decisions in epidemic emergency situations that may otherwise be driven by panic and influenced heavily by the media.

Received August 21, 2003. Accepted for publication February 27, 2004.

Acknowledgments: We thank the participants of the "Intolerable Burden of Malaria" pre-Multilateral Initiative on Malaria meeting, Arusha, Tanzania, 2002, for their useful comments following a presentation of an earlier version of this paper.

Financial support: Eve Worrall is a member of the Health Economics and Financing Program, which is funded by the United Kingdom Department for International Development (DFID). The United Kingdom DFID supports policies, programs, and projects to promote international development. It also provided funds for this study as part of that objective. The Roll Back Malaria Technical Support Network on malaria epidemics, managed by the World Health Organization, provides direction, advice, and financial support to prone/ at risk countries to conduct relevant field studies on epidemic prevention and control.

Disclaimer: The views and opinions expressed in this report are those of the author(s) alone.

Authors' addresses: Eve Worrall, Health Policy Unity, Department of Public Health and Policy, London School of Hygiene and Tropical Medicine, Keppel Street, London WC1E 7HT, United Kingdom, Telephone: 44-20-7927-2142, Fax: 44-20-7637-5391, E-mail: Eve.Worrall@lshtm.ac.uk. Aafje Rietveld, Malaria Control Department, World Health Organization, 20 Avenue Appia, CH-1211, Geneva 27, Switzerland, Telephone: 41-22-791-3753, Fax: 44-22-7914824, E-mail: rietvelda@who.int. Charles Delacollette, Malaria Control Department, World Health Organization, 20 Avenue Appia, CH- 
1211, Geneva 27, Switzerland, Telephone: 41-22-791-3745, Fax: 4422-791-4824, E-mail: delacollettec@who.ch.

\section{REFERENCES}

1. Kiszewski AE, Teklehaimanot A, 2004. A review of the clinical and epidemiological burdens of epidemic malaria. Am J Trop Med Hyg 71 (suppl 2): 128-135.

2. Najera JA, Kouznetsov RL, Delacollette C, 1998. Malaria Epidemics. Detection and Control, Forecasting and Prevention. Geneva: World Health Organization.

3. Kovats SR, Bouma MJ, Hajat S, Worrall E, Haines A, 2003. El Niño and health. Lancet 362: 1418-1489.

4. World Health Organization, 2001. Malaria Early Warning Systems: Concepts, Indicators and Partners. A Framework for Field Research in Africa. Geneva: World Health Organization. WHO/CDS/RBM, 80.

5. Snow RW, Craig MH, Deichmann U, Marsh K, 1999. Estimating mortality, morbidity and disability due to malaria among Africa's non-pregnant population. Bull World Health Organ 77: 624-640.

6. WHO AFRO, 1996. Proposal for Accelerating the Implementation of the Regional Malaria Control Strategy: WHO-AFRO. Geneva: World Health Organization.

7. World Health Organization, 2004. Prevention and Control of Malaria Epidemics: Forecasting, Prevention, Early Detection and Control - From Policy to Practice. Report of an Informal Consultation, Leysin, Switzerland. Geneva; World Health Organization.

8. Gabaldon A, 1946. Las razones de endemicidad y de epidemicidad: dos nuevas medidas de interes malariometrico. Tijeretazos Malaria 10: 10-18.

9. World Health Organization, 1999. The World Health Report 1999. Geneva: World Health Organization.

10. Pagnoni F, Delacollette C, 2002. Clinical, Behavioural and SocioEconomic Factors Related to Severe Malaria-A Multicentre Study in the African Region. Harare, Zimbabwe: World Health Organization Regional Office for Africa.

11. World Health Organization, 2000. Management of Severe Malaria-A Practical Handbook. Geneva: World Health Organization.

12. Wickramasuriya, 1937. Malaria and Ankylostomiasis in the Pregnant Woman. Oxford, United Kingdom: Oxford University Press.

13. Hammerich A, Campbell OMR, Chandramohan D, 2002. Unstable malaria transmission and maternal mortality-experiences from Rwanda. Trop Med Int Health 7: 573-576.

14. Luxemburger C, Ricci F, Nosten F, Raimond D, Bathet S, White $\mathrm{N}, 1997$. The epidemiology of severe malaria in an area of low transmission in Thailand. Trans R Soc Trop Med Hyg 91: 256262.
15. Cot M, Brutus L, Pinell V, Ramoroson H, Raveloson A, Rabeson D, Rakotonjanabelo AL, 2002. Malaria prevention during pregnancy in unstable transmission areas: the highlands of Madagascar. Trop Med Int Health 7: 565-572.

16. Newman RD, Hailemariam A, Jimma D, Degifie A, Kebede D, Rietveld AEC, Nahlen B, Barnwell J, Steketee RW, Parise M, 2003. Burden of malaria during pregnancy in areas of stable and unstable transmission in Ethiopia during a non-epidemic year. J Infect Dis 187: 1765-1772.

17. Goodman CA, Mnzava AE, Dlamini SS, Sharp BL, Mthembu DJ, Gumede JK, 2001. Comparison of the cost and costeffectiveness of insecticide-treated bednets and residual housespraying in KwaZulu-Natal, South Africa. Trop Med Int Health 6: 280-295.

18. Guyatt HL, Kinnear J, Burini M, Snow RW, 2002. A comparative cost analysis of insecticide-treated nets and indoor residual spraying in highland Kenya. Health Policy Plann 17: 144-153.

19. Shulman CE, Dorman EK, Talisuna AO, Lowe BS, Nevill C, Snow RW, Jilo H, Peshu N, Bulmer JN, Graham S, Marsh K, 1998. A community randomized controlled trial of insecticidetreated bednets for the prevention of malaria and anaemia among primigravid women on the Kenyan coast. Trop Med Int Health 3: 197-204.

20. Dolan G, ter Kuile FO, Jacoutot V, White NJ, Luxemburger C, Malankirii L, Chongsuphajaisiddhi T, Nosten F, 1993. Bed nets for the prevention of malaria and anaemia in pregnancy. Trans $R$ Soc Trop Med Hyg 87: 620-626.

21. Shulman CE, Dorman EK, Cutts F, Kawuondo K, Bulmer JN, Peshu N, Marsh K, 1999. Intermittent sulphadoxinepyrimethamine to prevent severe anemia secondary to malaria in pregnancy: a randomised placebo-controlled trial. Lancet 353: 632-672.

22. Nosten F, ter Kuile FO, Maelankiri L, Chongsuphajaisiddhi T, Nopdonrattankoon L, Tangkitchot S, Boudreau E, Bunnag D, White NJ, 1994. Mefloquine prophylaxis prevents malaria during pregnancy: a double-blind, placebo-controlled study. $J$ Infect Dis 169: 595-603.

23. Roberts L, 2001. Mortality in Eastern Democratic Republic of Congo-Results from Eleven Mortality Surveys. New York: International Rescue Committee Health Unit.

24. Médicins sans Frontières, 2001. Violence and Access to Health in Congo (DRC), Results of Five Epidemiological Surveys. Brussels, Belgium: Médicins sans Frontières.

25. World Health Organization, 2002. Report of the Third Meeting of the Technical Support Network for Prevention and Control of Malaria Epidemics. Geneva: World Health Organization, December 10-11, 2001.

26. World Health Organization, 2003. A Practical Guideline for Prevention and Control of Malaria Epidemics in Africa (Draft). Geneva: World Health Organization. 